\title{
Highly Degenerated Ground States in Some Rings Modeled by the Ising Spins with Competing Interactions
}

\author{
W. Florek*, M. Antkowiak, G. Kamieniarz and K. Jaśniewicz-Pacer \\ Faculty of Physics, Adam Mickiewicz University in Poznań, Poland
}

\begin{abstract}
We discuss three Ising ring systems with competing interactions which are analogs of quantum systems and we show that they exhibit similar properties. In particular, the archetypal system of three antiferromagnetically coupled spins $s$ has two magnetically degenerated ground states with $|M|=s$, when $0<J_{13}=\alpha<1=J_{12}=J_{23}$. The same effect is observed in the centered rings and even-numbered systems with antiferromagnetic couplings between the second neighbors which are the geometrically frustrated.
\end{abstract}

DOI: 10.12693/APhysPolA.133.411

PACS/topics: 75.10.Jm, 75.50.Ee, 75.50.Xx

\section{Introduction}

The notion of frustration in spin systems was introduced in the spin glass theory [1-3], but recently it has been studied in quantum spin systems, especially in the context of magnetic molecules built of transition metal ions with local spin $s$ [4-12]. Kahn [15, 16] introduced a term "degenerate frustration" pointing out importance of the ground state (GS) degeneration in systems with competing interactions. On the other hand, studies of nonanuclear chromium molecules and their smaller analogues [5-10] not only have confirmed the Kahn results that the GS degeneration is present for a few welldetermined values of a Hamiltonian parameter, but have shown that in a certain domain of this parameter the GS total spin $S$ of geometrically frustrated spin system is the same as in the domain without the geometrical frustration. This specific region was assigned to the third type of frustration [5]. Some interesting results were obtained for quantum spin systems $[9,10]$. For example, in the case of systems with one "defect" bond there is a series of $2 s$ critical values of this coupling at which the GS total spin $S$ and its symmetry is changed. In this paper results for the Ising models are presented and it is shown that in such cases there is only one critical value (independent of a system size $n$ and a spin number $s$ ), so, qualitatively, the Ising spin systems resembles the Heisenberg model for quantum spins $s=1 / 2$.

Classical spin systems are geometrically frustrated if there exists at least one cycle of pairwise coupled spins $\left(s_{j_{1}}, s_{j_{2}}, \ldots, s_{j_{l}}, s_{j_{1}}\right)$ with an odd number of antiferromagnetic couplings $J_{j_{k} j_{k+1}}[1-3]$. This condition leads to a more general concept of the "energetic" frustration, what means that the ground state configuration (GSC) does not simultaneously satisfy all the constraints imposed by the Hamiltonian [17]. Usually, frustrated systems have highly degenerated GS. Some systems with

*corresponding author; e-mail: wojciech.florek@amu.edu.pl geometric and energetic frustration, but with the nondegenerated GS are discussed below. Moreover, the GSC's are the same as those realized in non-frustrated systems, so the third type of frustration is revealed. A paradigm example is provided by antiferromagnetic rings with odd number of spin carriers (corresponding to the $\mathrm{Cr}_{9}$ molecule and its analogues). The second model describes antiferromagnetic rings with even number of spins uniformly coupled to an additional spin $s_{0}$, related to $\mathrm{Fe}_{7}$ or $\mathrm{Gd}_{7}$ molecules $[18,19]$. At the end rings with competing interactions of the first and the second neighbors are discussed.

The Heisenberg interactions $\hat{\boldsymbol{s}}_{j} \cdot \hat{\boldsymbol{s}}_{k}$ are replaced by products of the $z$-components $s_{j}^{z} s_{k}^{z}$ for arbitrary values of spin numbers $s_{j}$. Energy of two coupled spins is $E_{j k}(\mu)=J_{j k} m_{j} m_{k}$, where $\mu=\left|m_{1}, \ldots, m_{n}\right\rangle,\left|m_{j}\right| \leqslant s$, $1 \leqslant j, k \leqslant n$, is the so-called Ising configuration. For $\bar{\mu}=\left|-m_{1}, \ldots,-m_{n}\right\rangle$ one has $E_{j k}(\bar{\mu})=E_{j k}(\mu)$, then there is always trivial two-fold magnetic degeneration (except for all $m_{j}=0$ ), which is neglected hereafter. Non-zero exchange integrals are antiferromagnetic with $J_{j k}=1$ or $J_{j k}=\alpha$ for any real number $\alpha$. Considered systems are homogeneous, so all $s_{j}=s$.

\section{Rings with a bond defect}

Energy of a configuration $\mu$ is given as $(n$ is an odd integer)

$$
E(\mu)=\sum_{j=1}^{n-1} m_{j} m_{j+1}+\alpha m_{n} m_{1}
$$

For $\alpha<0$ there is the unique GSC $\mu_{0}$ with $m_{j}=(-1)^{j-1} s$ and energy $E\left(\mu_{0}\right)=[(1-n)+\alpha] s^{2}$ (Fig. 1a); in this state magnetization $M(\mu)=\sum_{j=1}^{n} m_{j}=s$. When $\alpha>0$ the system is geometrically frustrated and for large enough $\alpha$ a pair of parallel spins has to be placed at one of the other $n-1$ bonds, so the degenerated GS contains, among others, $n-1$ "basic" configurations $\mu_{j}$ with $m_{j}=m_{j+1}$ for $1 \leqslant j<n$ and $|M|=s$ (Fig. 1b). For $s>1 / 2$ there are $(n-2)(2 s-1)$ "extra" GSC's $\mu_{k, m}$ with triples $\left(s, m_{k},-s\right), 1<k<n,\left|m_{k}=m\right|<s$, and $M=m$ (Fig 1c). All these $2 s(n-2)+1$ GSC's for $\alpha>1$ yield 


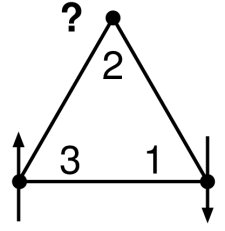

(a)

(c)

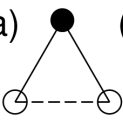

(b)

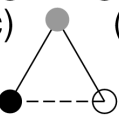

(d)
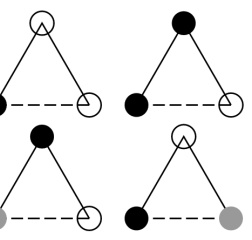

Fig. 1. Standard picture of the frustrated three-spin Ising system and actual ground state configurations of a system with a defect bond $\left(J_{12}=J_{23}=1, J_{13}=\alpha\right)$. (a) The unique configuration for $\alpha<1$; (b) $(n-1)$ "basic" configurations for $\alpha>1$; (c) $(n-2)(2 s-1)$ "extra" configurations for $\alpha>1$; (d) 2(2s-1) additional configurations at $\alpha=1$. The cases (c) and (d) are possible for $s>1 / 2$ only. Empty, black, and gray circles denote $m_{j}= \pm s$ and $\left|m_{j}\right|<s$, respectively.

the GS energy $E\left(\mu_{j}\right)=E\left(\mu_{k, m}\right)=[(3-n)-\alpha] s^{2}$. Both above discussed types of the GSC's are degenerated at $\alpha_{\mathrm{c}}^{\mathrm{DB}}=1, E=(2-n) s^{2}$, where $2(2 s-1)$ additional states, with $\left|m_{1}\right|<s$ or $\left|m_{n}\right|<s$ have the same energy, so the total degeneration at $\alpha=1$ equals $2 n s$ (Fig. 1d).

\section{Centered rings}

For an even number $n$, spins $s_{j}, 1 \leqslant j \leqslant n$, are placed at vertexes of a regular polygon. They are coupled to the nearest neighbors, $J_{j j+1}=\alpha$, and uniformly to an extra spin $s_{0}, J_{0 j}= \pm 1$, so $(n+1 \equiv 1)$

$$
E(\mu)=\alpha \sum_{j=1}^{n} m_{j} m_{j+1}+\varepsilon m_{0} M_{R}, \quad M_{R}=\sum_{j=1}^{n} m_{j},(2)
$$

where $\varepsilon= \pm 1$; this system is geometrically frustrated for $\alpha>0$. For $\alpha<0$ the ring is ordered ferromagnetically, with $M_{R}=n s$, and $m_{0}=-\varepsilon s$ (Fig. 2a). So, the GS en- (a)

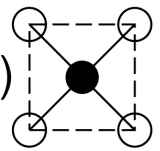

(b)
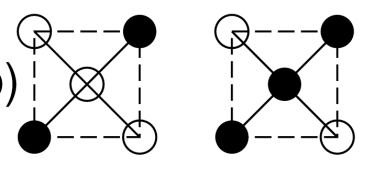

(c)
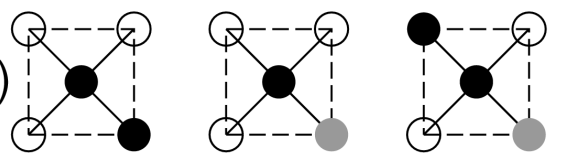
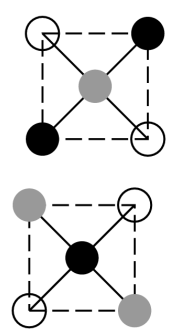

Fig. 2. Ground state configurations for the Ising-like spins $s_{j}, 1 \leqslant j \leqslant 4$, placed at vertexes of a square and $s_{0}$ at its center $\left(J_{j+1}=\alpha, J_{0 j}=1\right)$. (a) The unique configuration for $\alpha<1 / 2$; (b) $(2 s+1)$ configurations for $\alpha>1 / 2$; (c) four types of additional configurations at $\alpha=1 / 2$. Empty, black, and gray circles denote $m_{j}= \pm s$ and $\left|m_{j}\right|<s$, respectively. The sign of $m_{0}$ has to be changed for $J_{0 j}=-1$.

ergy equals $n(\alpha-1) s^{2}$. For large values of $\alpha$ the first term dominates and the ring is ordered antiferromagnetically, $m_{j}=(-1)^{j-1}$ for $1 \leqslant j \leqslant n$. Then $M_{R}=0$ and the total energy, $-n \alpha s^{2}$, does not depend on $m_{0}$ and $\varepsilon$. So the degeneration of this GS equals $(2 s+1)$ (Fig. $2 \mathrm{~b}$ ). These two types of GS's are degenerated at $\alpha_{\mathrm{c}}^{\mathrm{CR}}=1 / 2$. Moreover, at this point many additional configurations enter the GS. Their number can be determined from combinatorial considerations, but this problem is left out in this paper. For $n=4$ there are $8 s(s+1)-2$ such configurations (Fig. 2c), so the total degeneration at $\alpha=1 / 2$ amounts to $8 s^{2}+10 s$.

\section{Second neighbors}

Even-numbered rings become geometrically frustrated when antiferromagnetic interactions of the next-nearest neighbors are present. In the simplest, uniform, case the energy is given as $(n+p \equiv p)$

$$
E(\mu)=\sum_{j=1}^{n}\left(m_{j} m_{j+1}+\alpha m_{j} m_{j+2}\right) .
$$

The second term describes two antiferromagnetic "subrings", which are geometrically frustrated when $n / 2$ is an odd number. For $\alpha<0$ the non-frustrated system has the unique antiferromagnetic GS with $E\left(\mu^{\mathrm{AF}}\right)=$ $n(\alpha-1) s^{2}$ (Fig. 3a). Large $\alpha>0$ should lead to an- (a)

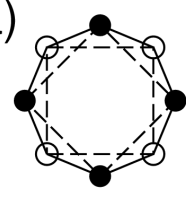

(b)

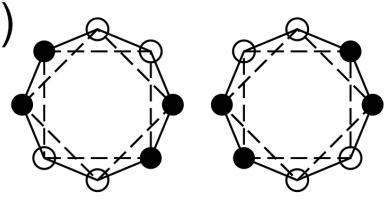

(c)

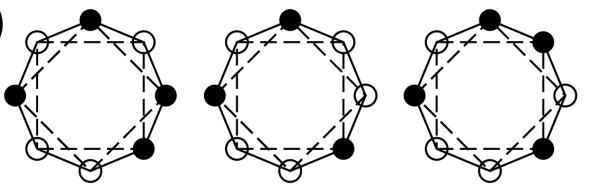

Fig. 3. Ground state configurations for the Ising-like spins $s_{j}=1 / 2,1 \leqslant j \leqslant 8$, placed at vertexes of a regular octagon $\left(J_{j+1}=1, J_{j+2}=\alpha\right)$. (a) The unique configuration for $\alpha<1 / 2$; (b) two configurations for $\alpha>1 / 2$; (c) three typical additional configurations at $\alpha=1 / 2$. Empty and black circles denote $m_{j}= \pm 1 / 2$, respectively.

tiferromagnetically ordered subrings. However, this is possible for $n / 2$ even only, so the cases $n=4 q$ and $n=4 q+2$ are discussed separately. In the first case, there are two non-equivalent antiferromagnetic configurations of subrings, so the two-fold degenerated GS comprises $\mu^{\mathrm{AF} 1}=|s, s,-s,-s, \ldots,-s,-s\rangle$ and $\mu^{\mathrm{AF} 2}=$ $|s,-s,-s, \ldots,-s,-s, s\rangle$ (Fig. 3b). Hence, the GS energy equals $E\left(\mu^{\mathrm{AF} 1(2)}\right)=-n \alpha s^{2}$. Comparing this value with the previous result one obtains the critical value $\alpha_{\mathrm{c}}^{\mathrm{SN}}=1 / 2$. Additional states at $\alpha=1 / 2$ enter the GS for $n>4$ and, for example, there 20 such states (so 23 in total) for $n=8$ and $s=1 / 2$ (Fig. 3c). Note that presented configurations have $M=0$ in the first two cases, whereas at $\alpha=1 / 2$ some of them have $M \neq 0$.

For $n=4 q+2$ (and large enough $\alpha>0$ ), the subrings are geometrically frustrated and their configurations are degenerated, what increase the whole system GS degeneration in comparison with the previous case. The minimum energy amounts to $[(4-n) \alpha-2] s^{2}$ and the same 

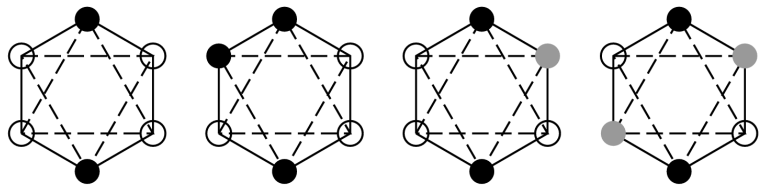

Fig. 4. Ground state configurations for the Ising-like spins $s_{j}=1,1 \leqslant j \leqslant 6$, placed at vertexes of a regular hexagon $\left(J_{j j+1}=1, J_{j+2}=\alpha>1 / 2\right)$. Four typical configurations are presented. Empty, black, and gray circles denote $m_{j}= \pm 1$ and $m_{j}=0$, respectively.

critical value $\alpha_{\mathrm{c}}^{\mathrm{SN}}=1 / 2$ is obtained. For $\alpha>1 / 2$ degeneration of the GS equals $n s[(n-4) s+2]$ (Fig. 4). There is no additional configurations at $\alpha=1 / 2$ for $n=6$, but degeneration at this point increases rapidly for $n \geqslant 10$.

\section{Summary}

Three systems discussed above show some common features. At first, the parameter $\alpha$ has the critical value $\alpha_{\mathrm{c}}>0$, independent on the system size $n$ and the spin number $s$. When $\alpha<\alpha_{\mathrm{c}}$ the GS is not degenerated, except for the trivial change $m_{j} \rightarrow-m_{j}$ for all spins $s_{j}$. It has to be stressed that for $0<\alpha<\alpha_{\mathrm{c}}$ the systems considered are geometrically (so also energetically) frustrated, but the degenerate (Kahn) frustration is absent. Moreover, these systems retain appropriate GS's from the range $\alpha<0$, where the geometrical frustration is absent. In other words, despite the presence of competing interactions ( $J$ and $J^{\prime}$, say) the systems considered do not change their GSC's if the appropriate ratio $\alpha=\left|J^{\prime}\right| /|J|$ is small enough, i.e. $\alpha<\alpha_{\text {c }}$. Therefore, all of them exhibit the third type of frustration in this domain. Analogous behavior has been found in the classical and quantum counterparts of models discussed here $[9,10,20]$. However, in each of these cases systems behave in different ways when the ratio $\alpha$ is large enough. The classical spin vectors $\boldsymbol{s}_{j}$ start to change their positions (they rotate in planar systems what leads to two configurations with different chirality). In the quantum systems the GS, which is a linear combination of many Ising configurations $\mu$, is modified in a continuous way except for a series of well-determined "critical" values of $\alpha$, when the GS is significantly changed. In both systems, the critical values are size- and spin-dependent. The Ising-like model shows the unique critical value with highly degenerated GS when $\alpha \geqslant \alpha_{\mathrm{c}}$.

\section{References}

[1] G. Toulouse, in: Spin Glass Theory, Beyond, Eds. M. Mézard, G. Parisi, M.A. Virasoro, World Sci., Singapore 2004, p. 99; reprinted from Comm. Physique 2, 115 (1997).
[2] J. Vannimenus, G. Toulouse, J. Phys. C: Solid State Physics 10, L537 (1977).

[3] S. Kirkpatrick, Phys. Rev. B 16, 4630 (1977).

[4] Y. Furukawa, K. Kiuchi, K. Kumagai, Y. Ajiro, Y. Narumi, M. Iwaki, K. Kindo, A. Bianchi, S. Carretta, P. Santini, F. Borsa, G.A. Timco, R.E.P. Winpenny, Phys. Rev. B 79, 134416 (2009).

[5] M.L. Baker, G.A. Timco, S. Piligkos, J.S. Mathiesone, H. Mutka, F. Tuna, P. Kozłowski, M. Antkowiak, T. Guidi, T. Gupta, H. Rath, R.J. Woolfson, G. Kamieniarz, R.G. Pritchard, H. Weihe, L. Cronin, G. Rajaraman, D. Collison, E.J.L. McInnes, R.E.P. Winpenny, Proc. Nat. Acad. Sci. 109, 19113 (2012).

[6] P. Kozłowski, M. Antkowiak, G. Kamieniarz, J. Nanopart. Res. 13, 6093 (2011).

[7] M. Antkowiak, P. Kozłowski, G. Kamieniarz, G.A. Timco, F. Tuna, R.E.P. Winpenny, Phys. Rev. B 87,184430 (2013).

[8] P. Kozłowski, Phys. Rev. B 91, 174432 (2015).

[9] G. Kamieniarz, W. Florek, M. Antkowiak, Phys. Rev. B 92, 140411(R) (2015).

[10] W. Florek, M. Antkowiak, G. Kamieniarz, Phys. Rev. B 94, 224421 (2016).

[11] G. Kamieniarz, R. Matysiak, W. Florek, S. Wałcerz, J. Mag. Mag. Mater. 203, 271 (1999).

[12] G. Kamieniarz, P. Kozłowski, G. Musiał, W. Florek, M. Antkowiak, M. Haglauer, A. Caramico D'Auria, F. Esposito, Inorg. Chim. Acta 361, 3690 (2008).

[13] E. Garlatti, S. Bordignon, S. Carretta, G. Allodi, G. Amoretti, R. De Renzi, A. Lascialfari, Y. Furukawa, G.A. Timco, R. Woolfson, R.E.P. Winpenny, P. Santini, Phys. Rev. B 93, 024424 (2016).

[14] M. Sobocińska, M. Antkowiak, M. Wojciechowski, G. Kamieniarz, J. Utko, T. Lis, Dalton Trans. 45, 7303 (2016).

[15] O. Kahn, Chem. Phys. Lett. 265, 109 (1997).

[16] J. Schnack, Dalton Trans. 39, 4677 (2010).

[17] H.T. Diep, H. Giacomini, in: Frustrated Spin Systems, Ed. H.T. Diep, World Sci., Singapore 2013, p. 1.

[18] A.M. Ako, O. Waldmann, V. Mereacre, F. Klöwer, I.J. Hewitt, Ch.E. Anson, H.U. Güdel, A.K. Powell, Inorg. Chem. 46, 756 (2007).

[19] J.W. Sharples, D. Collison, E.J.L. McInnes, J. Schnack, E. Palacios, M. Evangelisti, Nature Commun. 5, 5321 (2014).

[20] M. Grajek, T. Kopyciuk, W. Florek, A. Marlewski, Acta Phys. Pol. A 133, 417 (2018). 